\title{
Investigation of Endurance Performance of Carbon Nanotube Cathodes
}

\author{
By Nanako SAITO ${ }^{1)}$, Yoshiki YAMAGIWA ${ }^{1)}$, Yasushi OHKAWA ${ }^{2)}$, Shin-ichiro NISHIDA ${ }^{2)}$, Shoji KITAMURA ${ }^{2)}$ \\ ${ }^{1)}$ Graduate School of Engineering, Shizuoka University, Hamamatsu, Japan \\ ${ }^{2)}$ Aerospace Research and Development Directorate, Japan Aerospace Exploration Agency, Tokyo, Japan
}

(Received May 2nd, 2008)

\begin{abstract}
The Aerospace Research and Development Directorate of the Japan Aerospace Exploration Agency (JAXA) is considering a demonstration of electrodynamic tether (EDT) systems in low Earth orbit (LEO). Carbon nanotubes (CNTs) have some advantages as electron sources compared to conventional Spindt type emitters, and so are expected to be useful in EDT systems. Experiments to investigate the durability of CNT cathodes in a space environment had been conducted in a diode mode, but it was found that electron extraction tests, in which the cathode with a gate electrode is used, are necessary to evaluate the endurance of CNTs more accurately. In this paper, we conducted long duration operating tests of a cathode with a gate. It was found that there was almost no change in cathode performance at current densities below $100 \mathrm{~A} / \mathrm{m}^{2}$ even after the cathode was operated for over 500 hours in the high vacuum environment.
\end{abstract}

Key Words: Carbon Nanotubes, Field Emitters, Electrodynamic Tether, LEO Environment

\section{Nomenclature}

$\begin{array}{ll}E & \text { :electric field } \\ r_{\text {tip }} & \text { :radius of curvature of a tip } \\ d & \text { :electrode separation } \\ V & \text { :interelectrode voltage } \\ J & \text { :current density } \\ e & \text { :electron charge } \\ h & \text { :Plank's constant } \\ \phi & \text { :work function } \\ m_{e} & \text { :electron mass }\end{array}$

\section{Introduction}

The excellent field emitter properties of carbon nanotubes (CNTs) are expected to make them useful as electron emitters in electrodynamic tether (EDT) systems. The Aerospace Research and Development Directorate of the Japan Aerospace Exploration Agency (JAXA) has been conducting research and development of CNT cathodes for EDT systems ${ }^{1,2)}$.

An EDT system comprises a conductive tether with plasma contactors attached to each end. Electrons are collected from the ambient plasma at one end and are emitted at the other, forming a closed circuit through the tether and the ambient plasma. The interaction between the current flowing through the tether and the geomagnetic field generates a Lorentz force on the tether. Three electron emitter technologies are usually considered for space applications: hollow cathode, thermionic cathode and field emission cathode (FEC). It is believed that FECs are the most suitable for EDT systems because they have a very simple structure and are expected to operate with low power. A CNT cathode is a type of FEC.

Compared to Spindt type cathodes which have been conventionally studied as FECs, CNT cathodes are expected to have high efficiency, stable operation, and long life in the low earth orbit (LEO) atomic oxygen and plasma environment. However, their operational characteristics and the degradation mechanism of CNT cathodes in the space environment have not been proven so far. In this study, long duration operational tests of CNT cathodes were conducted at various current densities.

\section{Variety and Structures of CNTs}

Carbon nanotubes are roughly classified into single walled CNTs (SWNTs) and multiwalled CNTs (MWNTs). An SWNT is a graphite sheet rolled into a cylinder of a few micrometers in length and a few nanometers in diameter. An MWNT consists of several such cylinders nested inside each other ${ }^{3,4)}$. An SWNT has a higher emission efficiency than an MWNT because of its sharpness. On the other hand, the MWNT's multiple layers give it greater mechanical strength and so it is expected that MWNTs will have a longer life.

\section{Field Emission Cathodes}

\subsection{Fundamentals of FECs}

Figure 1 shows (a) a typical example of a Spindt type cathode, which consists of a gate electrode and an array of cone type metallic emitters, and (b) a single element of the cathode. Generally, the gate is a metal film with apertures over each emitter.

FECs are electron sources using the phenomenon of field emission of electrons from the surface of a solid material. The electric field concentrates at the emitter tips and when a voltage is applied between the gate and the emitters, electrons are emitted by the tunnel effect, as shown in Fig. 1(b). 
(a)

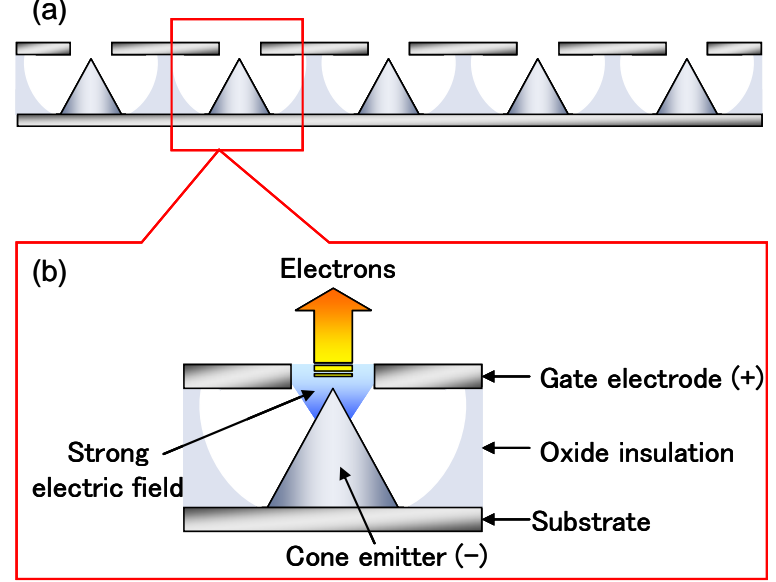

Fig. 1. (a) Schematic of Spindt type cathode, (b) a single element of the cathode.

Here, we briefly introduce the fundamentals of field emission using the model shown in Fig. 2, in which a single emitter with a parabolic shaped tip faces a simple disk anode.



Fig. 2. Model of a single parabolic shaped emitter facing an anode.

The electric field $E$ obtained by applying a voltage between the emitter and the anode can be written as follows ${ }^{5)}$ :

$$
E=\frac{2 V}{r_{\text {tip }} \ln \left(2 d / r_{\text {tip }}\right)}
$$

where $r_{\text {tip }}$ is the radius of curvature of the tip, $d$ is the electrode separation, and $V$ is the interelectrode voltage. The current density $J$ obtained by field emission is given by the Fowler-Nordheim (F-N) equation in Eq. (2) ${ }^{5)}$

$$
J=\frac{e^{3} E^{3}}{8 \pi h \phi} \exp \left(-\frac{8 \pi \sqrt{2 m_{e}}}{3 h e E} \phi^{3 / 2}\right)
$$

where $e$ is the electron charge, $h$ is Planck's constant, $\phi$ is the work function of the emitter, and $m_{e}$ is the electron mass. From Eq. (1) and (2), it can be understood that the electron emission capability strongly depends on the sharpness of the radius of curvature of the tip and the work function of the emitter.

\subsection{Advantages and Disadvantages of FECs}

For Spindt type cathodes, it is possible to arrange the emitter array uniformly to obtain a homogeneous current distribution, as shown in Fig. 1(a). However, the array's performance is degraded by two effects. If ambient ions or ions generated between the electrodes sputter the tips, the resulting increase in tip radius reduces the geometrically enhanced electric field at the tips and field emission capability degrades rapidly. Also, the work function increases due to the formation of an oxide film on the emitter surface due to reaction with atomic oxygen ${ }^{6}$. From Eq. (2), an increase in the work function means a greater voltage is needed to extract electrons, which causes a degradation of cathode performance.

In contrast, CNT cathodes have the drawback of a non-uniform current distribution because it is difficult to align each nanotube uniformly. On the other hand, CNTs have high mechanical strength and are highly resistant to ion bombardment: even though ion bombardment abrades the nanotube tips, it is expected that tip sharpness is retained because of the tubular shape of the CNT. Moreover, CNTs are expected to be more durable than Spindt type emitters because their surfaces are believed to be chemically stable and inert. However, their tolerance to atomic oxygen and oxygen plasma in LEO has not been sufficiently verified at the present time.

\section{Review of Previous Works ${ }^{7)}$}

JAXA has conducted experiments to evaluate the environmental durability of CNT cathodes. Since the objective was merely to evaluate durability, the experiments were conducted in a diode mode, which consists of a CNT emitter and an anode without a gate electrode.

First, operating tests were performed in various gaseous atmospheres to investigate the influence of gas species. While ions generated by electron bombardment in the vicinity of the cathode were found to have almost no effect on cathode performance, oxygen caused irrecoverable degradation. We believe that the chemisorption of oxygen on the CNT surface increases the work function and so increases the operating voltage.

Next, endurance tests were conducted in an oxygen environment to study cathode degradation characteristics. The results showed a gradual degradation at emission current densities below $100 \mathrm{~A} / \mathrm{m}^{2}$, but in the case of $260 \mathrm{~A} / \mathrm{m}^{2}$, the operating voltage rose rapidly. It was supposed that when current density increases to a certain level, the temperature of the CNT becomes high enough to cause chemisorption of oxygen on its surface and evaporation of the CNT itself due to oxidation. In some cases, the cathode suddenly lost its electron emission capability. We supposed that multiple micro-discharges occurred between the anode and emitter, causing CNTs to be removed from the emitter surface. Factors causing micro-discharge were considered to be gases emitted from the anode and CNTs and insufficient inter-electrode separation.

From these results, it was supposed that CNT cathode 
degradation and failure was closely related to heat radiation and outgassing from the anode. Consequently, it was suggested that endurance tests should be performed in an electron extraction condition, which is equivalent to practical emitter operation. These experiments are the focus of this paper.

\section{Experimental Apparatus}

Figure 3 shows the schematic of the experimental setup and the configuration of the CNT cathode used in the new experiments. To simulate practical operating conditions and remove anode influences, we used a cathode consisting of a gate, a mask and a CNT emitter, and covered these with a shield case. Electrons are extracted by the gate and collected by an anode placed opposite to the cathode body. The mask on the emitter surface plays an important role in preventing electrons from flowing into the gate ${ }^{1)}$.

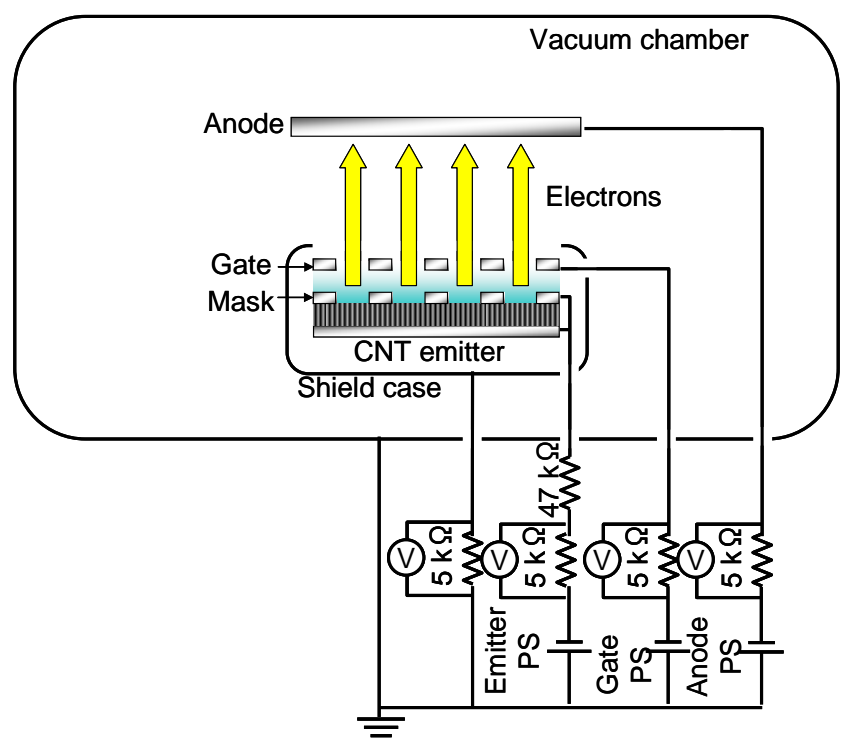

Fig. 3. Schematic of the experimental setups.

Figure 4 shows (a) the appearance of the cathode and (b) a close-up view of the double slit of the mask and gate. Figure 4(b) shows that the gate and mask have overlapping slit apertures. The physical characteristics of the mask, gate and anode are summarized in Table 1.

The current to the emitter, gate, mask, and case are measured across four $5 \mathrm{k} \Omega$ shunt resistances using analog input boards. The bias voltages of the emitter, gate, and anode are controlled automatically by analog output boards.
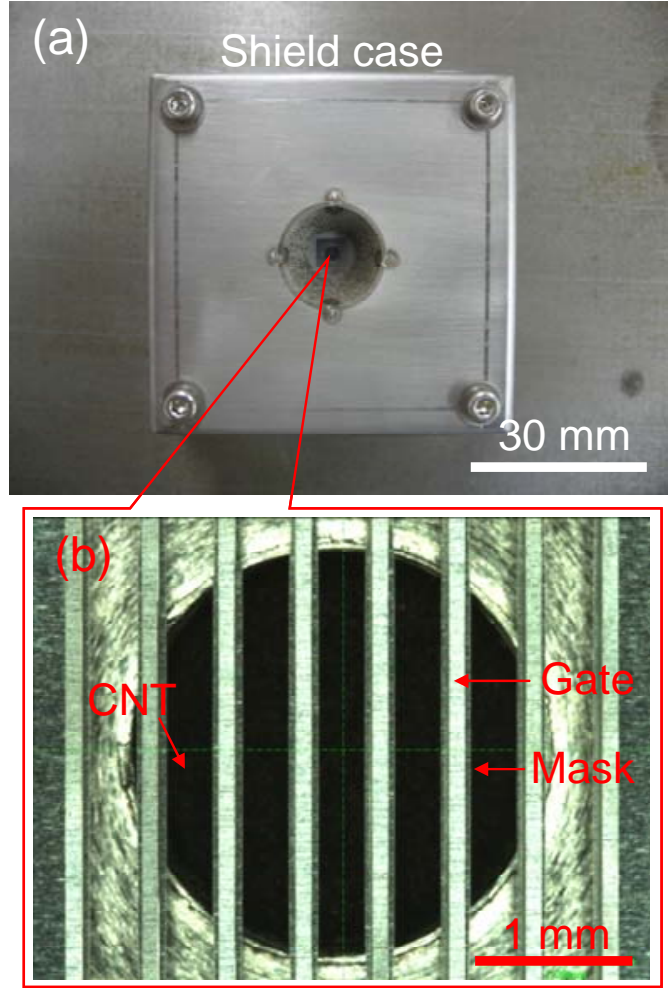

Fig. 4. (a) Appearance of the cathode, (b) close-up view of the double slit of the mask and gate.

Table 1. Mask, gate and anode physical characteristics.

\begin{tabular}{|l|c|c|c|}
\hline & Mask & Gate & Anode \\
\hline Material & Mo & Mo & SUS \\
\hline Thickness[mm] & 0.05 & 0.1 & 3.0 \\
\hline Slit width[mm] & 0.4 & 0.4 & \\
\hline Rib width[mm] & 0.1 & 0.1 & \\
\hline
\end{tabular}

Figure 5 shows detail of a CNT emitter. The emitter is a stainless substrate coated with a CNT film of numerous MWNTs grown by arc discharge. The CNTs are a few tens of micrometers in height and a few nanometers in diameters. Figure 6 shows a SEM image of the CNT film bonded to the substrate. As can be seen, the CNTs used in our study are complexly intertwined.

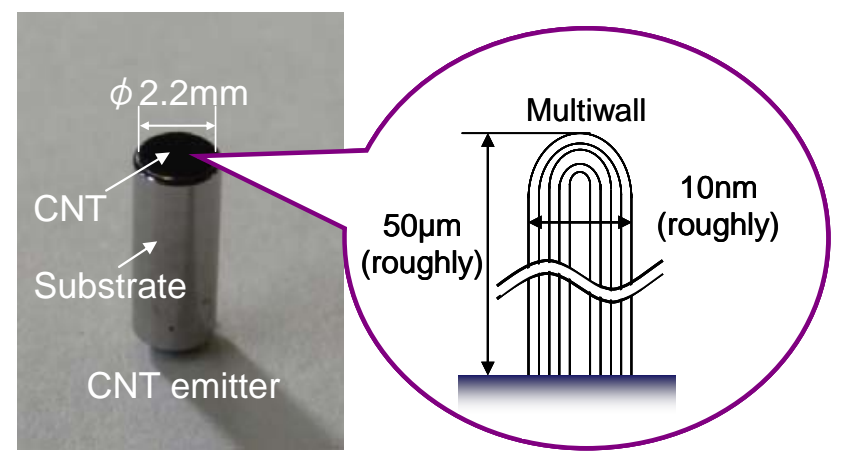

Fig. 5. Detail of a CNT emitter. 




Fig. 6. SEM image of a CNT emitter surface.

The cathode was set in vacuum chamber which could be evacuated to a pressure on the order of $10^{-6} \mathrm{~Pa}$. Figure 7 shows typical current-voltage characteristics of the CNT cathode obtained at a chamber pressure of approximately $1.5 \times 10^{-5} \mathrm{~Pa}$. The emitter current indicates the total current emitted from CNTs and is equivalent to the sum of the current intercepted by the gate, case and the anode current. Current density is calculated by dividing each current by the surface area of the CNT emitter including the area covered by the mask. In this experiment, the gate and anode voltages were fixed at $0 \mathrm{~V}$ and $100 \mathrm{~V}$, respectively, and the emitter bias voltage was increased by $1 \mathrm{~V}$ per second until the emission current density reached $70 \mathrm{~A} / \mathrm{m}^{2}$. The instantaneous values of current and emitter potential were recorded at 10 -second intervals. The emission current measurement error is less than $4 \%$, which includes the error of measurement systems and the fluctuating characteristics of the emission current. Figure 7 shows that the electron current flow to the gate is negligible. This high extraction efficiency is attributable to the beam converging effect by the mask on the emitter.

Fig. 7. Current-voltage characteristics of a CNT cathode with a gate.

\section{Results and Discussion}



Two long duration operating experiments were conducted in high-vacuum conditions at various current densities to investigate the operating characteristics of a CNT cathode with a gate. Unless noted otherwise, current density refers to emitter current. Electron extraction voltage indicates the emitter-gate voltage.

In the first experiment, the current density was varied between 50 and $100 \mathrm{~A} / \mathrm{m}^{2}$ and the electron extraction voltage to sustain each current density was measured. The test conditions are summarized in Table 2. A single emitter sample was used in all the cases. The current density was first set at $50 \mathrm{~A} / \mathrm{m}^{2}$ for 170 hours, then was controlled at $75 \mathrm{~A} / \mathrm{m}^{2}$ for 65 hours, $50 \mathrm{~A} / \mathrm{m}^{2}$ for 70 hours, $75 \mathrm{~A} / \mathrm{m}^{2}$ for 20 hours, $100 \mathrm{~A} / \mathrm{m}^{2}$ for 160 hours, and finally $50 \mathrm{~A} / \mathrm{m}^{2}$ for 30 hours by turns.

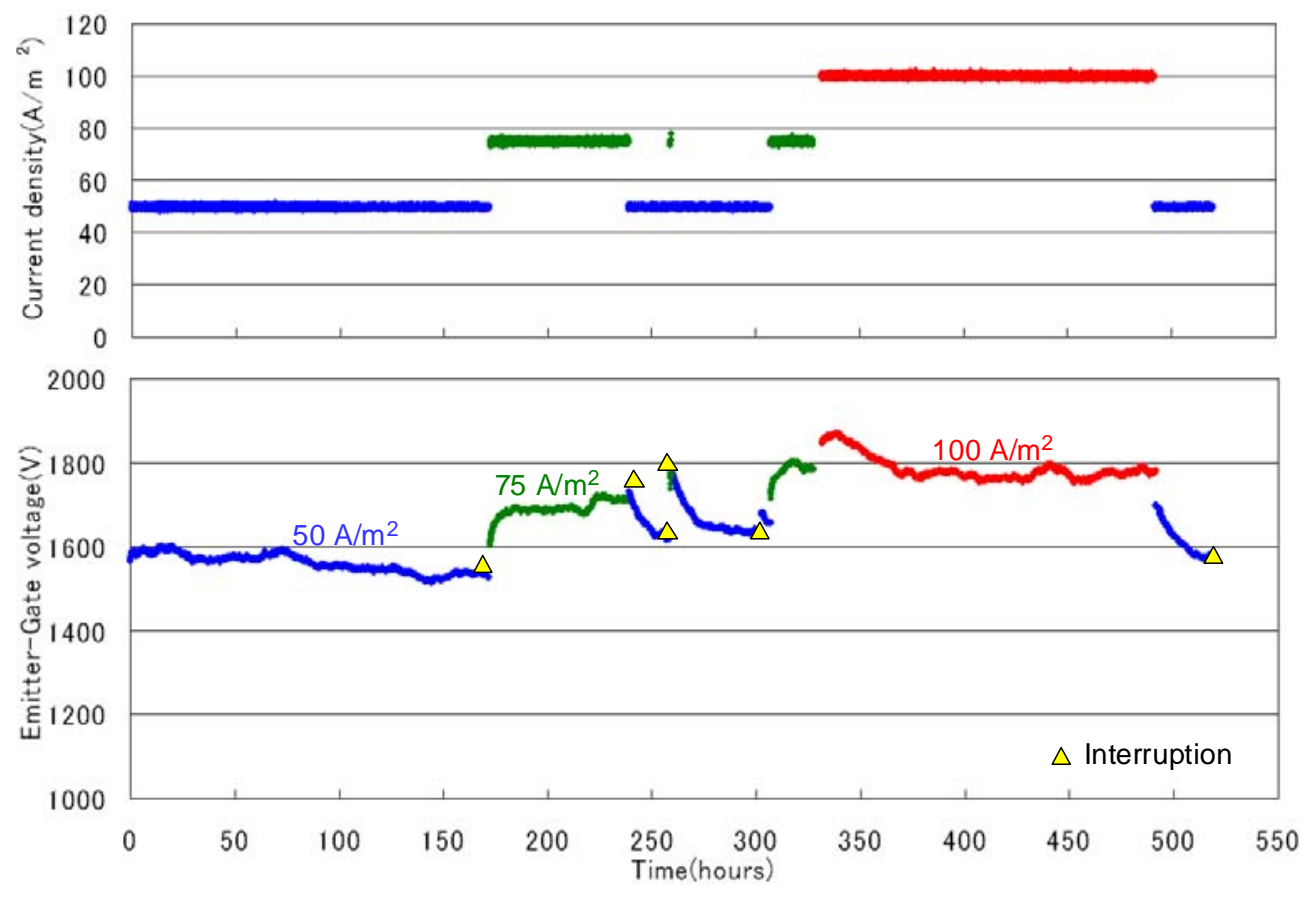

Fig. 8. Historical logs of current density (top) and emitter-gate voltage (bottom). 
Table 2. Conditions of the first experiment

\begin{tabular}{|l|l|}
\hline Pressure & $8.8 \times 10^{-6}-1.2 \times 10^{-5} \mathrm{~Pa}$ \\
\hline Emitter-gate distance & $0.4 \mathrm{~mm}$ \\
\hline Emitter-anode distance & $15 \mathrm{~mm}$ \\
\hline Emitter potential & $-100 \mathrm{~V}$ \\
\hline Gate potential & Positive bias \\
\hline Anode potential & $100 \mathrm{~V}$ \\
\hline Current density & $50,75,100 \mathrm{~A} / \mathrm{m}^{2}$ \\
\hline
\end{tabular}

Figure 8 shows the historical logs of the emission current and the emitter-gate voltage needed to maintain each current density. It is seen that the extraction voltage becomes stable within a few tens of hours after setting each current density of 50,75 , and $100 \mathrm{~A} / \mathrm{m}^{2}$.

After the CNT cathode had been operated for about 160 hours at $100 \mathrm{~A} / \mathrm{m}^{2}$, the current density was set to $50 \mathrm{~A} / \mathrm{m}^{2}$. A few tens of hours later, the electron extraction voltage decreased rapidly and recovered to a level comparable with the voltage when the operating test was started at $50 \mathrm{~A} / \mathrm{m}^{2}$. From this result, it is assumed that even though the cathode was operated for a few hundred hours at below $100 \mathrm{~A} / \mathrm{m}^{2}$, there was almost no effect on cathode performance due to CNT degradation in the high vacuum environment. Consequently, the elevation of the extraction voltage when current density was changed from 50 to $75 \mathrm{~A} / \mathrm{m}^{2}$ is a transitional phenomenon and is not attributable to CNT degradation.

In the second experiment, the current density was first maintained at $25 \mathrm{~A} / \mathrm{m}^{2}$. When the emitter-gate voltage had stabilized, $25 \mathrm{~A} / \mathrm{m}^{2}$ was added to the initial emission current. This process was repeated until the current density reached $100 \mathrm{~A} / \mathrm{m}^{2}$. Table 3 shows the experiment conditions. A single emitter sample was used in the experiment.

Table 3. Conditions of the second experiment.

\begin{tabular}{|l|l|}
\hline Pressure & $2.9 \times 10^{-6}-1.2 \times 10^{-5} \mathrm{~Pa}$ \\
\hline Emitter-gate distance & $0.3 \mathrm{~mm}$ \\
\hline Emitter-anode distance & $10 \mathrm{~mm}$ \\
\hline Emitter potential & Negative bias \\
\hline Gate potential & $0 \mathrm{~V}$ \\
\hline Anode potential & $475 \mathrm{~V}$ \\
\hline Current density & $25,50,75,100 \mathrm{~A} / \mathrm{m}^{2}$ \\
\hline
\end{tabular}

Figure 9 shows the historical logs of the emitter-gate voltage at each current density. In this figure, the time zero reference is when the cathode started to operate at each current density. The figure indicates that the extraction voltages tended to be almost stable throughout operation at all current conditions. This result agrees with that of the first experiment.

Before performing these two experiments, we had expected that the increases in voltage and the time required until the voltages stabilized would be proportional to the magnitude of the current density. However, no such tendency can be observed in Fig. 9. It seems that cathode performance in a high vacuum does not depend on the magnitude of the current density if the current density is below a certain level $\left(100 \mathrm{~A} / \mathrm{m}^{2}\right.$ in this case). Variation of surface conditions between different CNT emitter samples seems to be the more dominant factor affecting emission characteristics.

Further investigation is required to understand the

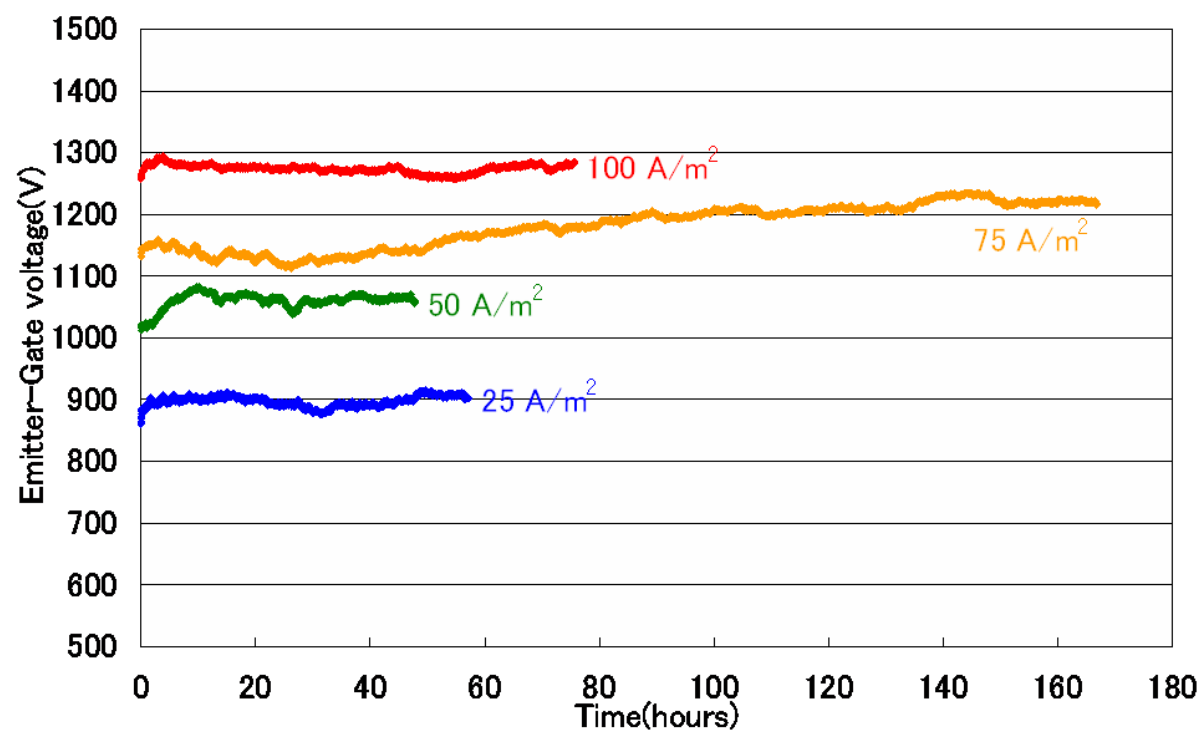

Fig. 9. Time-dependent variation of emitter-gate voltaSge at each current density. 
voltage variations seen in Fig. 9 in detail. We are planning approaches such as chemical analysis of the CNT emitter surface before and after operation, and in situ measurement of CNT temperature.

\section{Conclusion}

Long duration cathode operation tests were conducted using a cathode with a gate electrode to evaluate cathode endurance in conditions similar to those of operational electron extraction. Time-dependent characteristics of electron extraction voltages at various current densities were obtained. Almost no degradation of cathode performance was observed after a few hundred hours of operation in high vacuum conditions at current densities below $100 \mathrm{~A} / \mathrm{m}^{2}$.

We are now trying to simulate the LEO environment in which atomic oxygen is dominant, and to investigate the durability of CNT cathodes in an oxygen environment.

\section{References}

1) Okawa, Y., Kitamura, S., Kawamoto, S., Iseki, Y., Hashimoto, K., Noda, E.: An experimental study on carbon nanotube cathodes for electrodynamic tether propulsion, Acta Astronautica, 61/11-12 (2007), pp. 989-994.

2) Nakamura, Y., Yamagiwa, Y., Ohkawa, Y., Kawamoto, S., Nishida, S., Kitamura, S.: Environmental Effects on Electron Emission Characteristics of Carbon Nanotube Cathodes, $25^{\text {th }}$ ISTS, Kanazawa, ISTS 2006-s-18, 2006.

3) Niels de Jonge and Jean-Marc Bonard.: Carbon nanotube electron sources and applications, Phil. Trans. R. Soc. A 362 (2004), pp. 2239-2266.

4) http://www.surf.nuqe.nagoya-u.ac.jp

5) The Institute of Electrical Engineers of Japan: Electron and Ion Beam Engineering, Ohmsha, 1995 (in Japanese).

6) Marrese-Reading, C., Polk, J., and Jensen, K. L.: Experimental Performance Evaluations of $\mathrm{Mo}$ and $\mathrm{ZrC}$-Coated Mo Field Emission Array Cathodes in Oxygen Environment, IEPC Paper 01-278, 2001.

7) Saito, N., Nakamura, Y., Otsu, H., Yamagiwa, Y., Ohkawa, Y., Kitamura, S.: Investigation of Degradation and Failure Mechanism of Carbon Nanotube Cathodes, 51 ${ }^{\text {st }}$ Astronautical Science and Technology Conference, Sapporo, 3K14, 2007 (in Japanese). 